\title{
The Association Between Neighborhood Socioeconomic and Housing Characteristics with Hospitalization: Results of a National Study of Veterans
}

\author{
Elham Hatef, MD, MPH, Hadi Kharrazi, MD, PhD, Karin Nelson, MD, MSHS, \\ Philip Sylling, MA, Xiaomeng Ma, MS, Elyse C. Lasser, MS, Kelly M. Searle, PhD, \\ Zachary Predmore, AB, Adam J. Batten, BA, Idamay Curtis, BA, \\ Stephan Fibn, MD, MPH, and Jonathan P. Weiner, DrPH
}

Background: Social determinants of health (SDOH) have an inextricable impact on health. If remained unaddressed, poor SDOH can contribute to increased health care utilization and costs. We aimed to determine if geographically derived neighborhood level SDOH had an impact on hospitalization rates of patients receiving care at the Veterans Health Administration's (VHA) primary care clinics.

Methods: In a 1-year observational cohort of veterans enrolled in VHA's primary care medical home program during 2015, we abstracted data on individual veterans (age, sex, race, Gagne comorbidity score) from the VHA Corporate Data Warehouse and linked those data to data on neighborhood socioeconomic status (NSES) and housing characteristics from the US Census Bureau on census tract level. We used generalized estimating equation modeling and spatial-based analysis to assess the potential impact of patient-level demographic and clinical factors, NSES, and local housing stock (ie, housing instability, home vacancy rate, percentage of houses with no plumbing, and percentage of houses with no heating) on hospitalization. We defined hospitalization as an overnight stay in a VHA hospital only and reported the risk of hospitalization for veterans enrolled in the VHA's primary care medical home clinics, both across the nation and within 1 specific case study region of the country: King County, WA.

Results: Nationally, $6.63 \%$ of our veteran population was hospitalized within the VHA system. After accounting for patient-level characteristics, veterans residing in census tracts with a higher NSES index had decreased odds of hospitalization. After controlling all other factors, veterans residing in census tracts with higher percentage of houses without heating had 9\% (Odds Ratio, 1.09\%; 95\% CI, 1.04 to 1.14) increase in the likelihood of hospitalization in our regional Washington State analysis, though not our national level analyses.

Conclusions: Our results present the impact of neighborhood characteristics such as NSES and lack of proper heating system on the likelihood of hospitalization. The application of placed-based data at the geographic level is a powerful tool for identification of patients at high risk of health care utilization. (J Am Board Fam Med 2019;32:890-903.)

Keywords: Cohort Studies, Comorbidity, Housing Issues, Patient-Centered Care, Hospitalization, Population Health, Primary Health Care, Social Determinants of Health, Socioeconomic Status, United States Department of Veterans Affairs, Veterans Health, Washington

Social determinants of health $(\mathrm{SDOH})$ have an inextricable impact on health and wellness, with up to $40 \%$ of health outcomes being attributed to

This article was externally peer reviewed.

Submitted 12 April 2019; revised 29 July 2019; accepted 30 July 2019.
SDOH factors such as socioeconomic status (SES), housing issues, and food insecurity. ${ }^{1,2} \mathrm{SDOH}$ challenges can also contribute to increased health care 
utilization and costs. Thus, efforts addressing SDOH factors can potentially have a profound impact on life expectancy, healthier behaviors, and better overall health outcomes. ${ }^{3}$

Housing instability or subpar housing are among the most common SDOH in the United States. ${ }^{4,5}$ Housing issues such as lack of affordability or foreclosure and living in houses with substandard conditions could contribute to the development or exacerbation of serious health issues. $^{6-9}$ In addition, housing instability contributes to limited access to preventive health care, delayed filling of medication prescriptions, increased nonadherence to treatment plans, and consequently, higher utilization of acute health care resources. ${ }^{2,10,11}$ Housing issues do not represent isolated social problems that randomly distribute across neighborhoods. A number of inter-related socioeconomic conditions contribute to the development and worsening of housing problems. Hence, underserved populations are particularly vulnerable to experiencing housing issues resulting into poor health outcomes. ${ }^{12}$

Given the interconnected relationship of SDOH factors and health outcomes, health care providers are increasingly interested in developing interventions that fully consider the SDOH characteristics of their patients and neighborhoods. ${ }^{2}$ The Veterans Health Administration (VHA) as the largest integrated health system in the nation is committed to interventions that target key SDOH factors im-

(EH, HK, XM, ECL, KMS, ZP, JPW); Center for Health Disparities Solutions, Department of Health Policy and Management, Johns Hopkins Bloomberg School of Public Health, Baltimore, MD (EH); Division of Health Sciences Informatics, Johns Hopkins School of Medicine, Baltimore MD (HK); Veterans Affairs (VA) Puget Sound Health Care System, Seattle, WA (KN, PS, AJB, IC); Department of Medicine, University of Washington, Seattle, WA (KN, SF).

Funding: The Johns Hopkins University School of Public Health, Center for Population Health IT (CPHIT) performed this research under contract to the U.S. Department of Veterans Affairs as a case study which included data assessments and stakeholder input as necessary.

Conflict of interest: Any recommendations put forth in this paper are the views of the authors and do not necessarily represent the views of the U.S. Department of Veterans Affairs. Veterans Affairs (VA) had no disclosure of potential conflicts of interest.

Corresponding author: Elham Hatef, MD, MPH, Center for Population Health IT, Department of Health Policy and Management, Johns Hopkins Bloomberg School of Public Health, 624 N. Broadway, Room 502, Baltimore, MD 21205 (E-mail: ehatef1@jhu.edu). pacting the health of veterans and their communities. $^{13-15}$

To assist the VHA to achieve their goals in patient care and population health, we assessed whether geographically derived neighborhood socioeconomic status (NSES) and local housing stock had an impact on hospitalization rates of veterans receiving care at the VHA's primary care medical home clinics.

\section{Methods}

We linked patient-level demographic and clinical data from the VHA's electronic health record (EHR) system ${ }^{16}$ and population-level SDOH data (ie, SES and housing issues) from the veteran's neighborhood to assess the association between these factors and the risk of hospitalization for veterans enrolled in the VHA's primary care medical home clinics, both across the nation and within 1 specific case study region of the country: King County, WA. We defined hospitalization as an overnight stay in a VHA hospital only. We also applied geographic information system methods to visualize the distribution of hospitalization, NSES, and housing issues in the neighborhoods where veterans reside in King County, WA. The Institutional Review Board of Johns Hopkins University approved this study.

\section{Data Sources}

We obtained data on hospitalizations in 2015 from veterans with at least 1 primary care clinic visit during the year and a unique identification number assigned by the VHA (a total of 5,441,043 veterans). We obtained patient-level information on age, sex, race, and their residence's census tract Federal Information Processing Standards code from the VHA's Corporate Data Warehouse, ${ }^{17}$ which incorporates various databases, including the VHA's EHR. ${ }^{16}$ To address the severity of medical conditions resulted in hospitalization we obtained Gagne comorbidity score from VHA's Corporate Data Warehouse. ${ }^{18}$ Gagne comorbidity score, a single numeric comorbidity score for predicting short- and long-term mortality, constructed by combining conditions in the Charlson and Elixhauser measures. The score was presented as a continuous variable with higher score predicted higher risk of death. ${ }^{18}$ 


\section{Selection of SDOH Factors}

Similar to other EHRs, VHA's EHR lacked patient-level data on SDOH. ${ }^{19-21}$ To address this issue, we assessed $\mathrm{SDOH}$ factors on a populationlevel and used NSES index ${ }^{22}$ calculated for all veterans using the US Census Bureau, American Community Survey (2011 to 2015, 5-year estimate $)^{23}$ data on a census tract level. The NSES index was a summary measure of 6 geographic-level census-based variables that linked to the census tract of a participant's residence. The measure variables included 1) percentage of adults aged 25 years or older with less than high school education, 2) percentage of men who are unemployed, 3) percentage of household incomes below the poverty level, 4) percentage of households receiving public assistance, 5) percentage of households with children in which the head of household is female, and 6) median household income. We calculated the NSES index by computing a Z-score for each variable by subtracting the population mean and then dividing it by the population standard deviation. We then subtracted the Z-scores for the first 5 components from that of the median household income and scaled the results to the 0 to 100 range. ${ }^{22}$ The NSES index was presented as a continuous variable with the higher values corresponded to higher SES. For instance, a census tract with an NSES index of 100 would present higher SES and less SDOH challenges comparing to a census tract with NSES index of 90. The NSES index was validated for VHA patient population in previous studies. ${ }^{14}$

Through a literature review on SDOH studies and with feedback from an expert panel of VHA's clinical providers and policy makers, we selected a group of variables as measures of neighborhood housing issues. We categorized housing issues in each neighborhood with regard to 1) Homelessness, 2) Housing Instability or Insecurity (ie, "percentage of households that moved across census tracts in the past year and had an income below 100\% poverty line"), and 3) Characteristics of Individual Buildings (ie, "percentage of houses with no plumbing" and "percentage of houses with no fuel-based heating" ${ }^{\prime 23}$ defined as the heating system of a building using gas from underground pipes serving the neighborhood, bottled, tank, or liquefied petroleum, electricity, fuel oil, kerosene, coal or coke, wood, solar energy, or other fuel).
We also measured "Home Vacancy Rate" (percentage of vacant houses in each census tract) as an indicator of characteristics of a neighborhood. In this latter category we excluded vacant houses that were for rent, rented but not occupied, for sale, sold but not occupied, for seasonal, recreational, or occasional use, and for migrant workers.

\section{Statistical Analysis}

National Analysis

We performed descriptive analyses for a series of factors potentially associated with hospitalization among VHA primary care patients in 2015. For multivariate analyses we applied logistic regression using generalized estimating equations (GEEs) models with robust variance. ${ }^{24}$ The GEE models had patient-level variables for age, sex, race, and the Gagne comorbidity score. ${ }^{18}$ Taking into account the characteristics of our population and the distribution of each variable (refer to Table 1) we included race as a binary variable in the models comparing the majority of veterans (ie, whites) with the rest. We also included Gagne comorbidity score as a binary outcome comparing those with a score above 0 to 0 or below 0 score. We included the NSES index and housing variables as group or cluster-level variables representing census tract level measurements. We followed the recommendations from the National Academy of Medicine to stratify risk of hospitalization using NSES index quartiles in the GEE models. $^{25}$

The GEE models adjusted for the effect of the geographically clustered data in the NSES index and housing variables when assessing the association between hospitalizations and patient and population-level characteristics. ${ }^{26}$ These models enabled us to assess the impact of housing issues in isolation and also in the context of individual characteristics of veterans and socioeconomic challenges of their neighborhoods across the nation.

\section{Sample Small Area Analysis}

To limit the effect of potential unmeasured environmental factors (eg, availability of transportation) we reassessed the association between neighborhood characteristics with hospitalization on a smaller geographic scale, which is more homogenous with regard to those environmental factors. We performed the GEE modeling and spatial exploratory data analysis in King County, WA, where the VHA's office for the national 
Table 1. The Descriptive Analysis of Factors Affecting Hospitalization Among Veterans at VHA Primary Care Clinics Across the United States and in King County, WA in 2015*

\begin{tabular}{|c|c|c|c|c|}
\hline \multirow[b]{2}{*}{ Hospitalization } & \multicolumn{2}{|c|}{ United States } & \multicolumn{2}{|c|}{ King County, WA } \\
\hline & Yes & No & Yes & No \\
\hline Number of Patients (\%) & $360,527(6.63)$ & $5,080,516(93.38)$ & $1087(5.84)$ & $17022(91.47)$ \\
\hline Age, Mean (SD) & $64.46(13.86)$ & $62.7(16.50)$ & $63.69(14.03)$ & $58.83(17.21)$ \\
\hline Sex, $(\%)$ & $336,617(93.40)$ & $4,681,449(92.15)$ & $1,003(92.27)$ & $15,545(91.32)$ \\
\hline Race, $(\%)$ & $246,558(68.39)$ & $3,695,482(72.74)$ & $749(69.42)$ & $12156(72.33)$ \\
\hline Gagne Comorbidity Score, Mean (SD) & $2.07(2.07)$ & $0.33(1.24)$ & $1.25(1.95)$ & $0.34(1.14)$ \\
\hline NSES index ${ }^{\dagger}$, Mean (SD) & $0.67(0.11)$ & $0.69(0.10)$ & $0.72(0.08)$ & $0.74(0.08)$ \\
\hline \multicolumn{5}{|l|}{ Housing issues (Median, Range) } \\
\hline Housing instability ${ }^{\ddagger}$ & \multicolumn{2}{|c|}{$2.55(0.00,100.00)$} & \multicolumn{2}{|c|}{$2.90(0.00,41.89)$} \\
\hline Home vacancy rate r $^{\S}$ & \multicolumn{2}{|c|}{$3.34(0.00,100.00)$} & \multicolumn{2}{|c|}{$1.77(0.00,9.56)$} \\
\hline \multicolumn{5}{|l|}{ Characteristics of the House } \\
\hline Percentage of houses with no plumbing & \multicolumn{2}{|c|}{$0.00(0.00,62.71)$} & \multicolumn{2}{|c|}{$0.00(0.00,6.74)$} \\
\hline Percentage of houses with no heating & \multicolumn{2}{|c|}{$0.00(0.00,95.28)$} & \multicolumn{2}{|c|}{$0.00(0.00,16.67)$} \\
\hline
\end{tabular}

SD, standard deviation.

*The demographic data had a high completeness rate with less than $1 \%$ of data missing among the entire veteran population. Percentages might not add to $100 \%$ due to rounding and the missing data.

${ }^{\dagger}$ The NSES index was a summary measure of six geographic-level census-based variables that linked to the census tract of a participant's residence. The higher values corresponded to higher socioeconomic status.

${ }^{\ddagger}$ Percentage of households that moved across census tracts in the past year and had an income below $100 \%$ poverty line.

${ }^{\S}$ Percentage of vacant houses in each census tract.

NSES, Neighborhood Socio-economic Status, VHA, Veterans Health Administration.

evaluation of the patient-centered medical home initiative is located.

For the spatial exploratory data analysis, we mapped the hospitalization rate for VHA primary care patients across census tracts in King County, WA. ${ }^{27}$ We converted hospitalization to a binary variable (ie, any hospitalization in a VHA facility in 2015) and aggregated the number of hospitalizations for each census tract to calculate the numerator. Since the spatial analysis was performed at a census tract level we used the number of veterans per census tract as the population-at-risk representing the denominator. Using the aforementioned numerators and denominators, we mapped the NSES index and housing variables across King County, WA for side-by-side comparison of hospitalization distribution across the county in relation to neighborhood characteristics. We categorized each variable in the figures into different groups using the distribution of that variable. Such categorization made it easier to follow the color coding in each figure and to compare census tracts with different values. We considered a $P$-values $<.01$ as significance level. We conducted all analyses in $\mathrm{R}$ version 3.3.1 (using the GEE package for model- ing) ${ }^{28}$ and used the ArcGIS software (Redlands, CA) to generate the maps.

\section{Results \\ Assessment of Hospitalization among VHA Population}

The 2015 hospitalization rate was $6.63 \%$ for all veterans who enrolled in VHA primary care clinics and had at least 1 primary care clinic visit during the year. Most of the hospitalized veterans were male $(93.40 \%)$ and white $(68.39 \%)$. The mean age of hospitalized veterans was 64.46 years. Table 1 presents the results of the descriptive analysis for the entire VHA primary care population nationally and those resided in King County, WA.

Table 2 presents the GEE modeling for veterans across the US and those in King County, WA. Without considering patient-level characteristics and NSES factors, housing variables together mostly increased the odds of hospitalization for the national veterans population and those residing in King County, WA (Model 1). For instance, 1\% increase in the percentage of households with housing instability in the census tracts of veterans' residence resulted in $3 \%$ and $2 \%$ increased odds of 


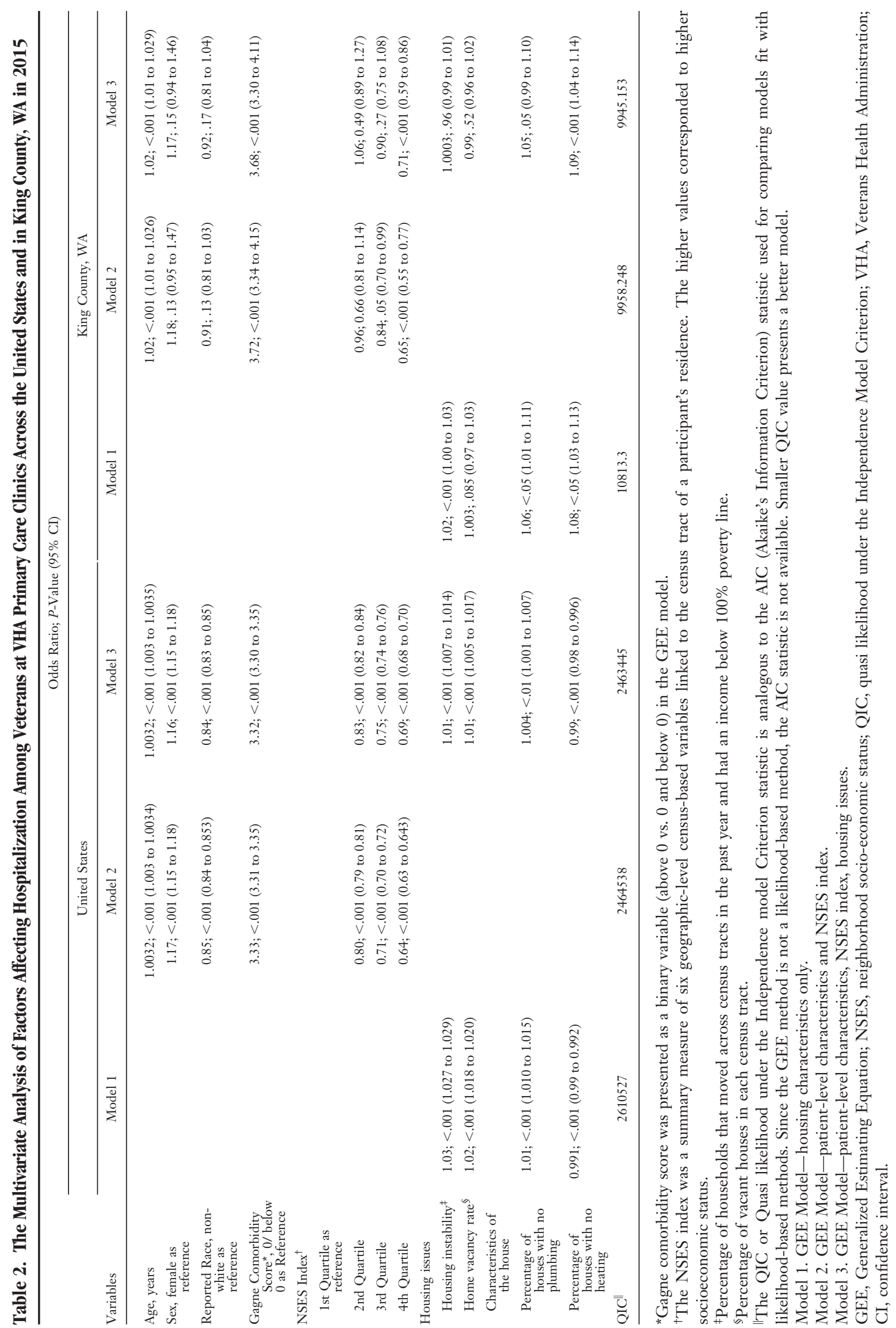


hospitalization for veterans across the US and those in King County, WA respectively and the results were statistically significant. However, measures of substandard housing characteristics (eg, lack of plumbing and fuel-based heating) had a stronger impact on odds of hospitalization when assessed in the smaller geographic region. Specifically, 1\% increase in the percentage of houses with no plumbing and fuel-based heating in the census tracts of veterans' residence in King County, WA resulted in $5 \%$ and $9 \%$ increased odds of hospitalization respectively, but the results were not statistically significant for houses without plumbing ( $P$-values of $<.05$ and $<.001$, respectively).

In terms of patient-level characteristics, male veterans across the country and those residing in King County, WA had a $17 \%$ and $18 \%$ increased odds of hospitalization compared with female veterans after accounting for NSES (Model 2) and a $16 \%$ and $17 \%$ increased odds of hospitalization after accounting for NSES and housing issues (Model 3) but the odds ratios (ORs) were not statistically significant for King County, WA $(P$ value $=.13$ and .15 , respectively). White veterans across the country and those residing in King County, WA had a $15 \%$ and $9 \%$ decreased odds of hospitalization compared with nonwhite veterans after accounting for NSES (Model 2) and a 16\% and $8 \%$ decreased odds of hospitalization after accounting for NSES and housing issues (Model 3). But the ORs were not statistically significant for King County, WA $(P$-value $=.13$ and .17 , respectively).

After accounting for patient-level characteristics, veterans residing in census tracts with a higher NSES index (ie, higher SES) had a decreased odds of hospitalization. For example, across the country and in King County, WA, veterans residing in census tracts with a second-quartile NSES index (higher NSES index compared with the first quartile) had respectively, a $20 \%$ and $4 \%$ decreased odds of hospitalization compared with those residing in census tracts with a first-quartile NSES index (Model 2). But the OR was not statistically significant for King County, WA $(P$-value $=.66)$. Adding housing issues in the model attenuated those odds. For instance, after accounting for housing issues across the country, veterans residing in census tracts with a second-quartile NSES index had a $17 \%$ decreased odds of hospitalization compared with those residing in census tracts with a first- quartile NSES index (Model 3). The odds of hospitalization decreased with each quartile increase of the NSES index for veterans across the country and in King County, WA with and without considering housing issues in the model (Models 2 and 3). But the ORs were only significant for the fourth quartile of the NSES index for veterans in King County, WA.

After accounting for patient-level characteristics and NSES of the neighborhoods, housing issues presented mixed associations with odds of hospitalization for veterans across the country and in King County, WA. For instance, $1 \%$ increase in the number of houses with no plumbing and fuel-based heating in the census tracts of veterans' residence in King County, WA resulted in 5\% and $9 \%$ increased odds of hospitalization (Model 3). But the results were not statistically significant for houses without plumbing $(P$-values of $<.05$ and $<.001$, respectively).

\section{Spatial Analysis}

Figure 1 illustrates the unadjusted hospitalization rate for the VHA population in 2015 across census tracts in King County, WA. The hospitalization rate was $18.43 \%$ and higher within several of the census tracts in the western border of the county; mostly in Seattle and its surrounding urban areas (Figure 1A, dark red). The same area also had a mean NSES index of 0.72 or lower, which represents a lower average SES in those neighborhoods (Figure 1B, light red and pink).

The mapping of housing issues across King County, WA shows spatial variation in housing instability rate (Figure 2A), home vacancy rate (Figure 2B), and percentages of houses with no plumbing (Figure 2C) or fuel-based heating (Figure 2D) across the county. Overall we detected census tracts with increased housing issues mostly scattered in the western border of the county (Figure 2, dark red).

\section{Discussion}

Overall, in our population the hospitalization rate of veterans within the VHA system was slightly lower than the national age-adjusted rate of overnight hospital stays $(6.63 \%$ vs $7.0 \%) .{ }^{29}$

\section{Impact of SDOH Variables}

Our multivariate modeling took into account a range of patient-level characteristics allowing us to 
Figure 1. Spatial analysis for Hospitalization and NSES Index. Map a: Unadjusted hospitalization rate and Map b: NSES mean per census tract in King County, WA. NSES: neighborhood socioeconomic status.
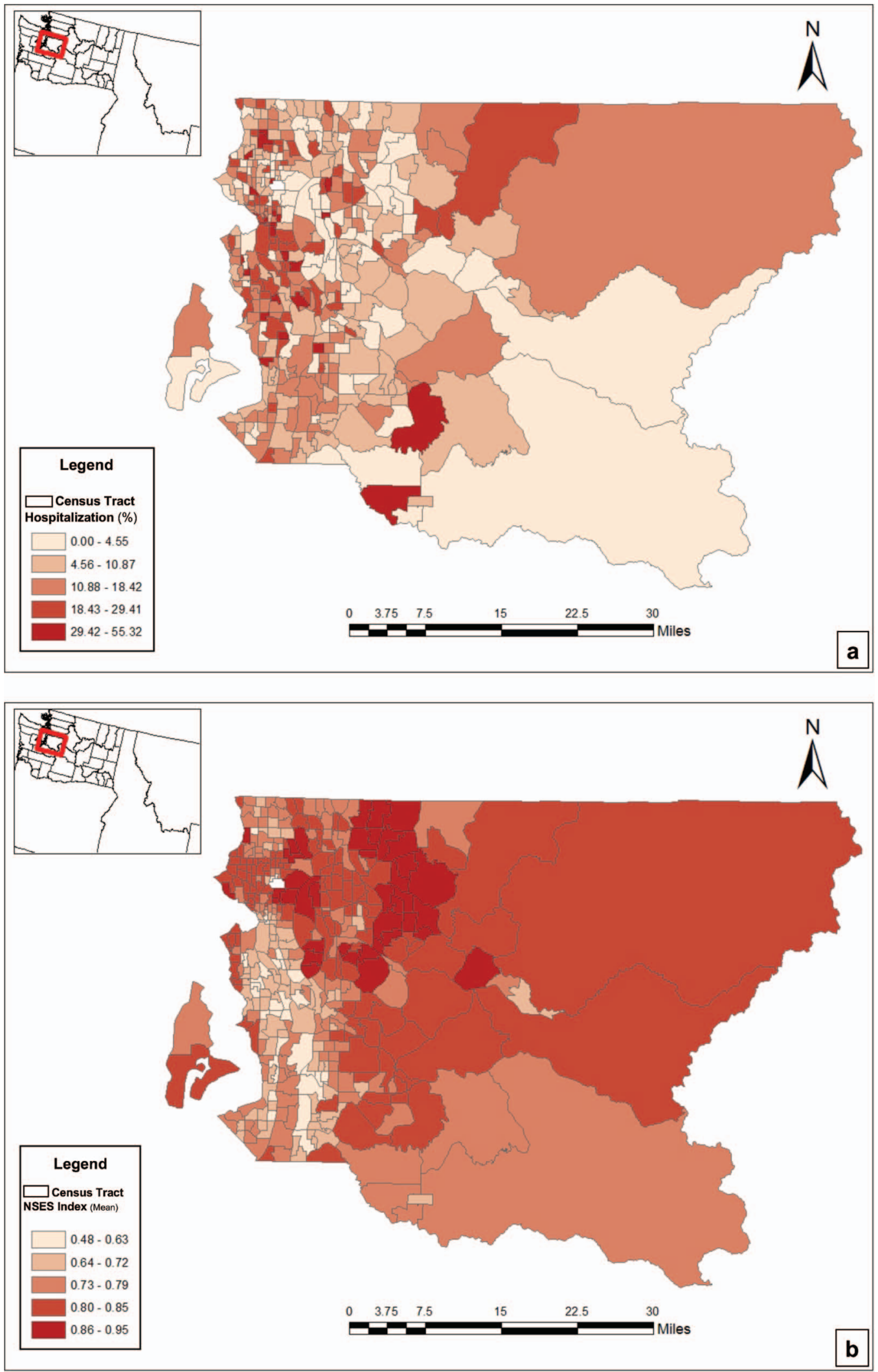
Figure 2. Spatial analysis for housing issues. Maps for (a) housing instability rate; (b) home vacancy rate; (c) percent of houses with no plumbing; (d) percentage of houses with no fuel-based heating.
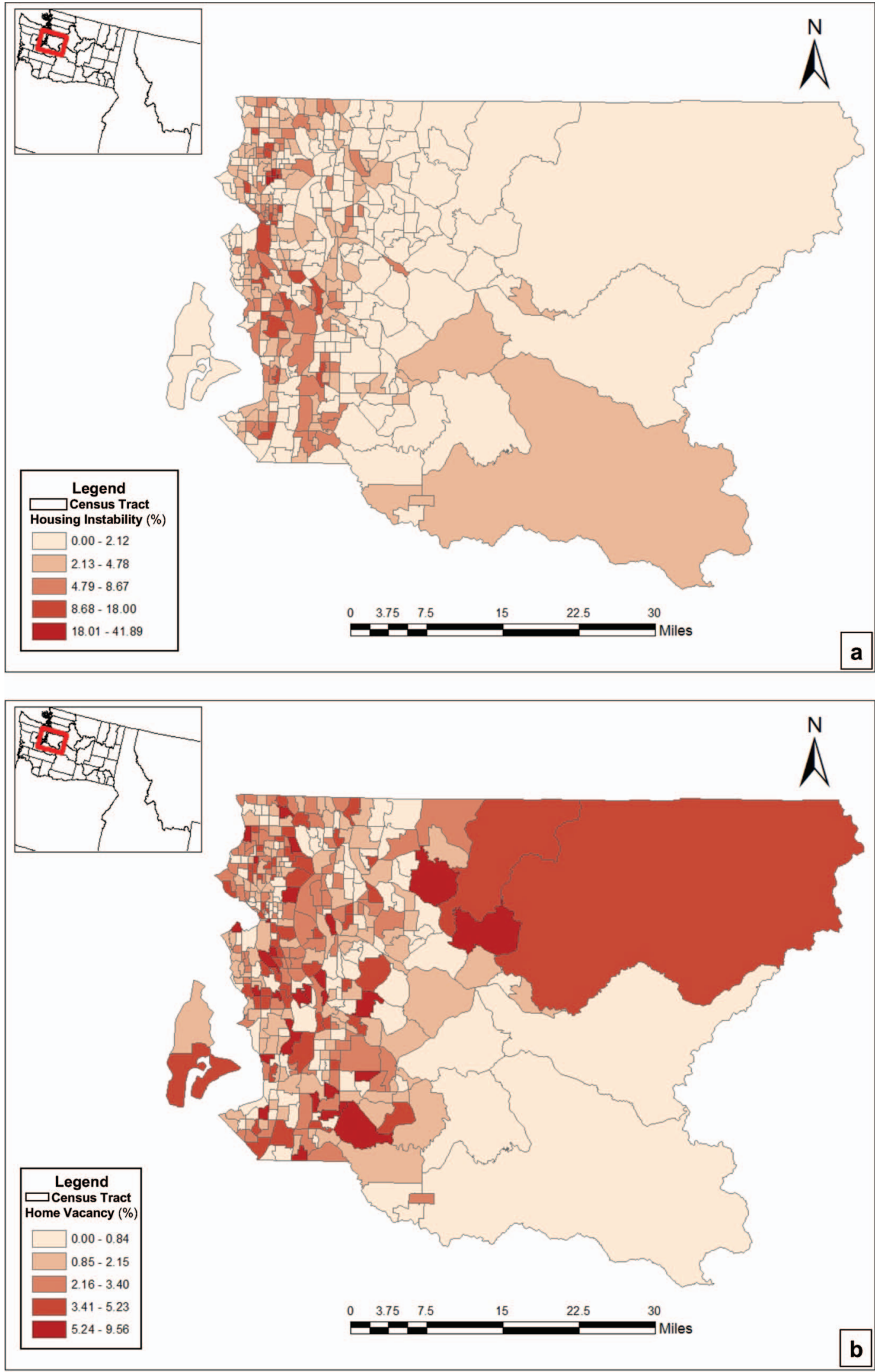
Figure 2. Continued.
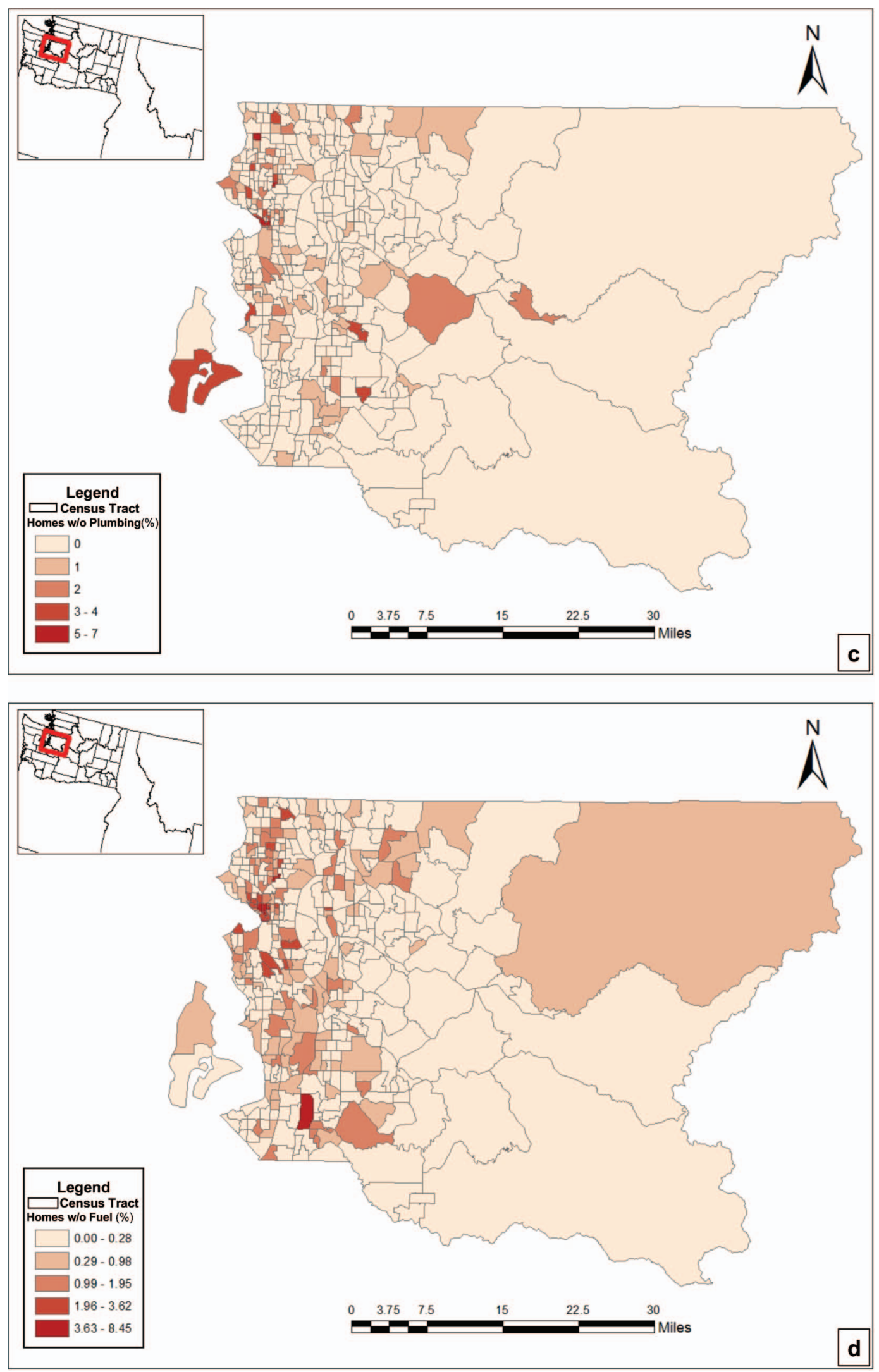
assess the impact of population-level characteristics on the census tract level for the entire VHA primary care population and within 1 specific case study region of the country; King County, WA. Model 1 findings were consistent with our descriptive analysis showing minimal association between the housing variables and the odds of hospitalization nationally and in King County, WA. However, when housing issues were assessed in the smaller region, holding other housing variables constant, there was some modest increase in hospitalization (from 6\% to $8 \%$ ) in neighborhoods with substandard housing with no plumbing or fuel-based heating.

Model 2 suggested that males and nonwhites had a slightly higher odds of hospitalization compared with females and whites nationally. But the ORs were not statistically significant in King County, WA. These results were in line with previous studies of the general population and those of the VHA population, for nonwhite veterans experiencing higher odds of being hospitalized..$^{30-32}$ In addition, Gagne comorbidity score of above 0 comparing to score 0 or below 0 conferred a 3 times higher odds of hospitalization and residing in neighborhoods with a higher SES score (represented by each progressively higher NSES quartile) resulted in a $20 \%$ to $36 \%$ decrease in the odds of hospitalization nationally and a $4 \%$ to $35 \%$ decrease in King County, WA.

The evidence varies on the impact of including SES data for assessment of the health outcomes. A number of prior studies found that including data on different social factors would improve prediction of health outcomes, such as hospitalization and readmission risk. ${ }^{30,31,33}$ In addition, SES data would narrow down the range of observed variations in readmission rates. ${ }^{34}$ In contrast, Bhavsar et $\mathrm{al}^{35}$ assessed the value of NSES in predicting the risk of adverse outcomes in EHR-based risk models. In their study, information on NSES did not contribute much more to risk prediction above and beyond demographic (eg, age and race) and insurance data already provided in the EHR.

In our study in the Model 3 for the national sample, the associations between demographic and clinical factors with the odds of hospitalization remained unchanged and the results of housing variables remained consistent, with Gagne comorbidity score having the largest OR and gender and race having larger ORs than housing variables. How- ever, assessing housing issues in the smaller region, while holding all other variables constant, revealed some modest increase in hospitalization (from 5\% to $9 \%$ ) in neighborhoods with substandard housing with no plumbing or fuel-based heating, but the results were not statistically significant for houses without plumbing. This indicates that substandard housing might have an impact on hospitalization when it is assessed in a smaller region and the effect of potential hidden environmental factors are limited.

Several of the ORs across 3 models were not statistically significant for King County, WA. We used census tract as the unit of analysis for population-level variables in the GEE models. There were 396 census tracts in King County, WA and the relatively small sample size might have played a role in nonsignificant ORs. Future studies should include population-level data at a lower granular level (eg, block groups), which would help to have a larger sample size and more homogenous population in each unit of analysis.

We did not identify studies assessing the impact of housing issues on hospitalization in a population similar to the one in this study using the housing variables that we included in our modeling. Instead, we compared our results with studies assessing the health-related impact of a wide range of housing issues and other socioeconomic characteristics of neighborhoods, using population-level data from US Census Bureau and other publicly available data sources. Our results deviated from those studies. For instance, Liu and Pearlman ${ }^{36}$ found that children living in neighborhoods with the highest proportion of crowded housing conditions, racial minority residents, or neighborhood-level poverty had higher hospital readmission rates. In addition, in Beck et al's ${ }^{37}$ study children living in neighborhoods with the highest quartile of housing code violations had higher odds of rehospitalization within 12 months, compared with those living in the lowest quartile. Gronlund et $\mathrm{al}^{38}$ revealed that the hospitalization rate related to extreme heat was higher in ZIP codes having older housing and in cities with lower air conditioning prevalence. Other neighborhood-level factors such as housing stress (defined as $30 \%$ or more of households living in a house that lacked complete plumbing and/or kitchen, renter or owner paid $30 \%$ or more of income for mortgage or rent, or had more than 1 person per room) have significantly impacted hos- 
pitalization. ${ }^{39}$ Lastly, living in newer and properly maintained buildings, neighborhoods with newer housing stock with no outward signs of physical deterioration, and buildings with a richer set of amenity features have decreased mental health care costs. ${ }^{40}$ The difference in our findings and those of other studies might reflect the different approach to development of variables addressing selected $\mathrm{SDOH}$ domains. It also indicates the necessity for development of standard quality measures for assessment of SDOH so that the results of different studies are comparable. In addition, our study population included all hospitalizations across VHA primary care population and did not include subgroup analysis of patients with specific clinical conditions such as asthma. Some SDOH factors might play a significant role in the health outcomes of specific patient populations (eg, housing issues and asthma).

\section{The Added Value of Spatial Analysis}

The data used in this study were unique and contained information on more than 5 million veterans during the year of study. Our access to the longitude and latitude of the patients' home location made it possible to link the patient-level data to the neighborhood characteristics. ${ }^{23}$ This geocoded data allowed the detection of spatial patterns for the entire study population. The geo-data also helped to identify neighborhoods with increased hospitalization rate across King County, WA, where hospitalization rate was much higher than the overall VHA population and the national rate (18.43\% and higher at several of the census tracts in the western border of the county mostly in the Seattle and its surrounding urban areas vs $6.63 \%$ for overall VHA population and 7.0\% nationally). ${ }^{29}$ The area corresponded to the neighborhoods containing several census tracts with a lower average SES, which would be a contributing factor in the high utilization as was presented in our models. Future studies with an in-depth assessment of other factors playing role in high hospitalization in these neighborhoods would help VHA to identify potential modifiable factors in these inner city neighborhoods. Similar interventions would be applicable to other inner city neighborhoods experiencing low SES and high health care utilization.

The geo-derived SDOH variables addressing housing issues had a low predictive power for hospitalization especially after incorporating robust
SES measures (eg, NSES index). However, using spatial analysis in the smaller geographic area enabled the identification of areas of high hospitalization rate that could then be mapped along with geoderived SDOH to identify where resources would be invested to improve behavior or the built environment. For example, areas with high hospitalization rate had neighborhoods with high vacancy rates or poor housing condition. This information would allow the VHA to develop interventions and focus resources to help veterans finding better housing. Performing the spatial analysis in a smaller geographic area also helped us to limit the effect of potential unmeasured environmental factors (eg, availability of transportation) in an area more homogenous with regard to those environmental factors.

\section{Study Limitations}

Our study had some limitations. The hospitalization data only reflected the overnight hospital stay in the VHA facilities but no other facilities. It did not distinguish between needed care due to acute or chronic health conditions and avoidable hospital utilization due to lack of access to other types of care. This secondary data analysis was limited to available patient-level data in VHA's EHR, which similar to most other EHRs did not include data on SDOH. ${ }^{19-21}$ Therefore, our assessment of SDOH was limited to population-level data and we did not assess whether any observed associations between $\mathrm{SDOH}$ and hospitalization were related to the composition of the area (ie, they served as proxies for individual factors) or whether they reflected the contextual factors of the area itself. For instance, we assessed the association between hospitalization and housing insecurity in a neighborhood as a proxy for housing insecurity of a veteran living in that neighborhood, but did not assess the association between hospitalization and the number of abandoned houses in the neighborhood as the contextual factor of the area itself. We did not include any variables to measure homelessness in our analysis, since the VHA has performed comprehensive assessment of veteran's homelessness status internally and US Census Bureau, American Community Survey ${ }^{23}$ did not include any data on homelessness.

Our NSES index was a robust measure of several key socioeconomic characteristics of the neighborhoods. The development of standard SDOH composite measures using both patient-level EHR and 
population-level SDOH data would help to take into account other $\mathrm{SDOH}$ domains such as housing characteristics to make a more comprehensive assessment of those factors and their interconnected impact on hospitalization. In addition, the linkage between patient-level health outcomes/utilization and population-level SDOH could be strengthen by examining individual screening responses to SDOH surveys and linking utilization as in the Accountable Health Communities initiative by the Innovation Center at the Centers for Medicare and Medicaid Services. ${ }^{41}$

The lack of a strong and significant impact for housing variables on hospitalization in our study might be due to the limited ability of a crosssection of data to present the long-term impact of SDOH factors on health outcomes and health care utilization. Assessment of this relationship in a longitudinal dataset with a longer time frame (5 to 10 years) might increase the impact of housing variables in predictive models for hospitalization. Several environmental factors (eg, availability of transportation) were not included in the national analysis due to lack of data availability in all locales, despite their likely effect on access to, and utilization of, services in primary care clinics across the country.

Nevertheless, our study presents the added value of neighborhood-level data such as those obtained by the US Census Bureau ${ }^{23}$ to data collected in EHRs and other clinical and administrative patient-level information systems, for identification of patients at risk of poor health outcomes and high social needs. Geographic variations in hospitalization rates based on demographic, clinical, socioeconomic, and housing factors could also signal differential access to care and disparities in quality of care, as has been shown in this study. ${ }^{32}$ The application of placed-based data to assess disparities at the geographic level or other population approaches in health care is a powerful tool for case management purposes of underserved populations. Using data available in small geographic areas can help in identifying those neighborhoods with low socioeconomic characteristics suffering from different social and health issues that require intervention and provide evidence to support health care policies and resource allocation to patients and communities in need. In addition, such visualization of data in small geographic areas provides an opportunity to convene local and regional stake- holders: philanthropy, social service providers, and health systems to address gaps in resources and capacity.

The authors thank our colleagues at the Department of Veterans Affairs Clinical Systems Development and Evaluation as well as Veterans Affairs Puget Sound Health Care System in Seattle, Washington for their support during this project.

To see this article online, please go to: http://jabfm.org/content/ 32/6/890.full.

\section{References}

1. Bachrach D, Pfister H, Wallis K, Lipson M. Addressing patients' social needs: An emerging business case for provider investment. 2014. Available from: https://www.commonwealthfund.org/sites/default/ files/documents/__media_files_publications_fund_ report_2014_may_1749_bachrach_addressing_ patients_social_needs_v2.pdf. Accessed April 8, 2019.

2. Health Research \& Educational Trust. Social determinants of health series: housing and the role of hospitals. Health Research \& Educational Trust. 2017. Available from: https://www.aha.org/ahahretguides/2017-08-22-social-determinants-healthseries-housing-role-hospitals. Accessed April 8, 2019.

3. National Prevention Council. National prevention strategy: America's plan for better health and wellness. U.S. Department of Health and Human Services, Office of the Surgeon General. 2011. Available from: https://www.surgeongeneral.gov/priorities/ prevention/strategy/report.pdf. Accessed April 8, 2019.

4. Root Cause Coalition. Housing and health: the connection, and innovative steps health systems are taking to address housing to improve health. The Root Cause Coalition. 2016. Available from: http://www. rootcausecoalition.org/wp-content/uploads/2016/ 11/White-Paper-Housing-and-Health.pdf. Accessed April 8, 2019.

5. Solari CD, Morris S, Shivji A, de Souza T. The 2015 annual homeless assessment report to congress, part 2: estimates of homelessness in the united states. U.S. Department of Housing and Urban Development, Office of Community Planning and Development. 2016. Available from: https://www.hudex change.info/onecpd/assets/File/2015-AHAR-Part-2. pdf. Accessed April 8, 2019.

6. Centers for Disease Control and Prevention. Most recent asthma data. Centers for Disease Control and Prevention Web Site. Available from: https://www. cdc.gov/asthma/asthmadata.htm. Accessed April 8, 2019.

7. Braveman P, Dekker M, Egerter S, Sadegh-Nobari T, Pollack C. Housing and health. Robert Wood Johnson Foundation. 2011. Available from: https:// 
www.rwjf.org/en/library/research/2011/05/housingand-health.html. Accessed April 8, 2019.

8. Downing J. The health effects of the foreclosure crisis and unaffordable housing: a systematic review and explanation of evidence. Soc Sci Med 2016;162: 88-96.

9. March E, Ettinger de Cuba S, Cook JT, et al. Behind closed doors: the hidden health impacts of being behind on rent. Children's HealthWatch. 2011. Available from: https://housingalliancepa.org/155p/. Accessed April 8, 2019.

10. Pollack CE, Griffin BA, Lynch J. Housing affordability and health among homeowners and renters. Am J of Prev Med 2010;39:515-521.

11. Kushel MB, Gupta R, Gee L, Haas JS. Housing instability and food insecurity as barriers to health care among low-income americans. J Gen Intern Med 2006;21:71-77.

12. Burgard SA, Seefeldt KS, Zelner S. Housing instability and health: findings from the Michigan recession and recovery study. Soc Sci Med 2012;75:22152224.

13. Wennberg DE, Sharp SM, Bevan G, Skinner JS, Gottlieb DJ, Wennberg JE. A population health approach to reducing observational intensity bias in health risk adjustment: Cross sectional analysis of insurance claims. BMJ 2014;348:g2392.

14. Nelson K, Schwartz G, Hernandez S, Simonetti J, Curtis I, Fihn SD. The association between neighborhood environment and mortality: results from a national study of veterans. J Gen Intern Med 2017; $32: 416-422$.

15. Lushniak BD, Alley DE, Ulin B, Graffunder C. The national prevention strategy: leveraging multiple sectors to improve population health. Am J Public Health 2015;105:229-231.

16. Brown SH, Lincoln MJ, Groen PJ, Kolodner RM. VistA-U.S. Department of Veterans Affairs national-scale HIS. Int J Med Inform 2003;69(2-3):135156.

17. U.S. Department of Veterans Affairs. Corporate data warehouse (CDW). Updated 2017. Available from: https://www.hsrd.research.va.gov/for_researchers/ vinci/cdw.cfm. Accessed April 8, 2019.

18. Gagne JJ, Glynn RJ, Avorn J, Levin R, Schneeweiss S. A combined comorbidity score predicted mortality in elderly patients better than existing scores. J Clin Epidemiol 2011;64:749-759.

19. Lindemann EA, Chen ES, Wang Y, Skube SJ, Melton GB. Representation of social history factors across age groups: a topic analysis of free-text social documentation. AMIA Annu Symp Proc 2018;2017: 1169-1178.

20. Winden TJ, Chen ES, Wang Y, Lindemann E, Melton GB. Residence, living situation, and living conditions information documentation in clinical practice. AMIA Annu Symp Proc 201812017:1783.

21. Winden TJ, Chen ES, Monsen KA, Wang Y,
Melton GB. Evaluation of flowsheet documentation in the electronic health record for residence, living situation, and living conditions. AMIA Jt Summits Transl Sci Proc 2018;2017:236-245.

22. Merkin SS, Basurto-Dávila R, Karlamangla A, et al. Neighborhoods and cumulative biological risk profiles by race/ethnicity in a national sample of U.S. adults: NHANES III. Ann Epidemiol 2009;19:194201.

23. The United States Census Bureau. American Community Survey (ACS). Available from: https://www. census.gov/programs-surveys/acs/. Accessed April 8, 2019.

24. Zeger SL, Liang KY. Longitudinal data analysis for discrete and continuous outcomes. Biometrics 1986; 42:121-130.

25. National Academies of Sciences, Engineering, and Medicine. Accounting for social risk factors in Medicare payment: Identifying social risk factors. Washington, DC: The National Academies Press. www.nap.edu/ $\operatorname{read} / 21858 /$ chapter/1. Published 2016. Accessed July 2019;21.

26. Collins TW, Grineski SE, Morales DX. Sexual orientation, gender, and environmental injustice: unequal carcinogenic air pollution risks in greater Houston. Ann Am Assoc Geogr 2017;107:72-92.

27. Kulldorff M. A spatial scan statistic. Communications in Statistics-Theory and Methods 1997;26: 1481-1496.

28. The $\mathrm{R}$ project for statistical computing. Available from: www.r-project.org. Accessed April 8, 2019.

29. National Center for Health Statistics. Centers for Disease Control and Prevention. Hospital utilization (in non-federal short-stay hospitals). Available from: https://www.cdc.gov/nchs/fastats/hospital.htm. Updated 2017. Accessed April 8, 2019.

30. Taylor LA, Tan AX, Coyle CE, et al. Leveraging the social determinants of health: What Works? PLoS One 2016;11:e0160217.

31. Calvillo-King L, Arnold D, Eubank KJ, et al. Impact of social factors on risk of readmission or mortality in pneumonia and heart failure: Systematic review. J Gen Intern Med 2013;28:269-282.

32. Krishnamurthi N, Francis J, Fihn SD, Meyer CS, Whooley MA. Leading causes of cardiovascular hospitalization in 8.45 million US veterans. PLoS One 2018;13:e0193996.

33. Hu J, Gonsahn MD, Nerenz DR. Socioeconomic status and readmissions: evidence from an urban teaching hospital. Health Aff (Millwood) 2014;33: $778-785$.

34. Nagasako EM, Reidhead M, Waterman B, Dunagan WC. Adding socioeconomic data to hospital readmissions calculations may produce more useful results. Health Aff (Millwood) 2014;33:786-791.

35. Bhavsar NA, Gao A, Phelan M, Pagidipati NJ, Goldstein BA. Value of neighborhood socioeconomic status in predicting risk of outcomes in studies that use 
electronic health record data. JAMA Network Open 2018; 1:e182716.

36. Liu SY, Pearlman DN. Hospital readmissions for childhood asthma: The role of individual and neighborhood factors. Public Health Reports (1974-) 2009;124:65-78.

37. Beck AF, Huang B, Chundur R, Kahn RS. Housing code violation density associated with emergency department and hospital use by children with asthma. Health Aff (Millwood) 2014;33:1993-2002.

38. Gronlund CJ, Zanobetti A, Wellenius GA, Schwartz JD, O'Neill MS. Vulnerability to renal, heat and respiratory hospitalizations during extreme heat among U.S. elderly. Climatic Change 2016;136:631645.

39. Xu S, Fortney JC, Dong F. Community-level correlates of hospitalizations for persons with schizophrenia. Psychiatr Serv 2009;60:772-778.

40. Harkness J, Newman SJ, Salkever D. The costeffectiveness of independent housing for the chronically mentally ill: do housing and neighborhood features matter? Health Serv Res 2004;39: 1341-1360.

41. Centers for Medicare and Medicaid Services. Accountable health communities model. Updated 2019. Available from: https://innovation.cms.gov/ initiatives/ahcm. Accessed July 7, 2019. 


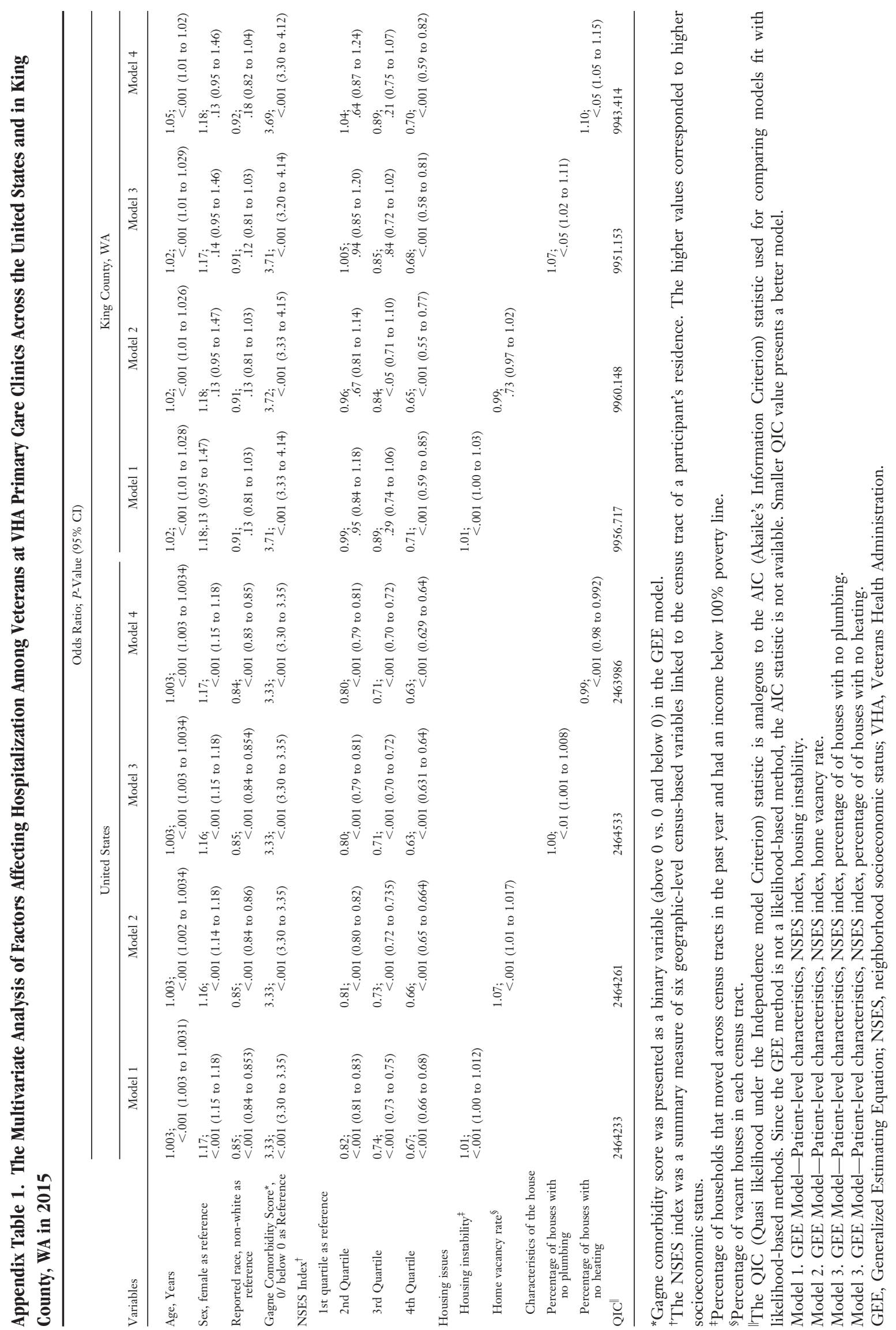


Appendix Table 2. Variance Inflation Factor Presented for the Multivariate Analysis of Factors Affecting Hospitalization Among Veterans at VHA Primary Care Clinics Across the United States and in King County, WA in 2015

\begin{tabular}{|c|c|c|c|c|}
\hline \multirow[b]{3}{*}{ Variables } & \multicolumn{4}{|c|}{ Variance Inflation Factor* (Degree of Freedom) } \\
\hline & \multicolumn{2}{|c|}{ United States } & \multicolumn{2}{|c|}{ King County, WA } \\
\hline & Model 1 & Model 2 & Model 1 & Model 2 \\
\hline Age & $1.068765(1)$ & $1.071784(1)$ & $1.068765(1)$ & $1.071784(1)$ \\
\hline Sex & $1.032169(1)$ & $1.032579(1)$ & $1.032169(1)$ & $1.032579(1)$ \\
\hline Reported Race & $1.053327(1)$ & $1.055482(1)$ & $1.053327(1)$ & $1.055482(1)$ \\
\hline Gagne Comorbidity Score & $1.014003(1)$ & $1.015308(1)$ & $1.014003(1)$ & $1.015308(1)$ \\
\hline NSES Index & $1.036799(3)$ & $1.438241(3)$ & $1.036799(3)$ & $1.438241(3)$ \\
\hline \multicolumn{5}{|l|}{ Housing Issues } \\
\hline Housing Instability & & $1.582049(1)$ & & $1.582049(1)$ \\
\hline Home Vacancy Rate & & $1.025891(1)$ & & $1.025891(1)$ \\
\hline \multicolumn{5}{|l|}{ Characteristics of the House } \\
\hline Percentage of houses with no plumbing & & $1.253458(1)$ & & $1.253458(1)$ \\
\hline Percentage of houses with no heating & & $1.248236(1)$ & & $1.248236(1)$ \\
\hline
\end{tabular}

*Variance Inflation Factor close to 1 presents least collinearity among variables in a model.

Model 1. GEE Model-Patient-level characteristics and NSES index.

Model 2. GEE Model-Patient-level characteristics, NSES index, and housing issues.

NSES, Neighborhood socioeconomic status; VHA, Veterans Health Administration. 